\title{
Genome sequence of plant associated rhizobacterium Bacillus amyloliquefaciens strain UCMB5033
}

\author{
Adnan Niazi, Shahid Manzoor, Sarosh Bejai, Johan Meijer, Erik Bongcam-Rudloff ${ }^{\bowtie}$ \\ Swedish University of Agricultural Sciences, Uppsala, Sweden
}

\section{Motivation and Objectives}

Over the past years, different measures have been adopted to increase crop production including chemical fertilizers and pesticides, and more recently genetic manipulation of plants. Such methods are costly tools for increasing the yield. In addition, side effects such as nutrient leakage, development of pesticide resistant strains, and negative effects on the environment call for other means to maintain food safety and security. Bacteria mediated bio-control is an alternative strategy that have great promise to overcome such problems. Bacillus amyloliquefaciens strain UCMB5033 is a spore forming Grampositive rhizobacterium that has shown great potential to serve as biocontrol agent. It has shown growth promotion and disease protection against insects and pathogens after developing symbiotic relationship with host plant; $A$. thaliana and oilseed rape $B$. napus (unpublished). Our ultimate goal is to eliminate the use of agrochemicals for production of crops. To achieve this, the aim of the study is to reveal and explain the genetic architecture that contributes to the plants ability to overcome biotic and abiotic stress based on bacterial bio-control. The availability and analysis of genome sequence will throw light on several biological aspects behind plant-bacterium interaction, such as plant colonization, priming, and stress tolerance in order to support durable plant protection and eliminate chemical pesticides.

\section{Methods}

The genome of B. amyloliquefaciens UCMB5033 was sequenced with Illumina multiplex technology and lon Torrent PGM systems. Whole genome assembly was accomplished by comparative assembly approach i.e. integrating both mapping and de novo assembly. The paired-end reads from Illumina were provided to MIRA v.3.4 (Chevreux et al., 1999) for mapping assembly and reads data from lon Torrent was assembled with Newbler v.2.8 by de novo assembly method
(Zerbino and Birney, 2008). Mapping assembly was performed against the available genome of B. amyloliquefaciens UCMB5036 (accession no. HF563562) (Manzoor et al., 2013). The contigs produced by Newbler assembler were moved according to the reference genome and aligned to the sequence obtained through mapping assembly, using Mauve genome alignment software (Darling et al., 2010). The genome sequence was annotated with a collection of annotation tools via Magnifying Genome (MaGe) Annotation Platform (Vallenet et al., 2009).

\section{Results and Discussion}

The combination of assemblies from reads data generated by two different NGS platforms contributed in speeding up the assembly process with high accuracy that resulted in B. amyloliquefaciens strain UCMB5033 assembled genome sequence. The genome sequence confirmed the presence of NRPS and PKS gene clusters: surfactin (srf), fengycin (fen), difficidin (dfn), bacilysin (bac), macrolactin ( $\mathrm{m} / \mathrm{n})$, bacillaene (bae), bacillomycin D (bmy), and bacillibactin (dhb) responsible for the synthesis of secondary metabolites, including antifungal and antibacterial compounds (Chen et al., 2007). Other genes involved in metabolism of plant derived compounds, resistance to drugs, root colonization and other functions that presumably give the bacterium an advantage in developing symbiotic relationship with plants were also present.

\section{Acknowledgements}

This work was supported by the grants from Swedish Research Council for Environment, Agricultural Sciences and Spatial Planning (FORMAS) and the Higher Education Commission (HEC), Pakistan. Sequencing was performed by the SNP\&SEQ Technology Platform, Science for Life Laboratory at Uppsala University, a national infrastructure supported by the Swedish Research Council (VR-RFI) and the Knut and Alice Wallenberg Foundation. Bioinformatics analysis 
were also supported by the BILS infrastructure at SLU.

\section{References}

Chen XH, Koumoutsi A, Scholz R, Eisenreich A, Schneider K et al. 2007. Comparative analysis of the complete genome sequence of the plant growth-promoting bacterium Bacillus amyloliquefaciens FZB42. Nature Biotechnol. 25:1007-1014. doi: 10:1038/n!̣t!1325

Chevreux B, Wetter T, Suhai S. 1999. Genomic sequence assembly using trace signals and additional sequence information. Computer Science and Biology: Proceedings of the German Conference on Bioinformatics (GCB) 99, pp. 45-56.
Darling AE, Mau B, Perna NT. 2010. progressiveMauve: multiple genome alignment with gene gain, loss and rearrangement. PLOS ONE 5:e11147. doi: 10.137]1/journal!: pone.0011147.

Manzoor S, Niazi A, Bejai S, Meijer J, Bongcam-Rudloff E. 2013. Bacillus amyloliquefaciens strain UCMB5036: The genome sequence of a plant associated bacterium. Genome Announc. 1(2): e00111-13. doi: 10,1128/genomeA.00111-13.

Vallenet $D$, Engelen S, Mornico D, Cruveiller S, Fleury L et al. 2009. MicroScope: a platform for microbial genome annotation and comparative genomics. Database (Oxford) 2009:bap021. doi: 101093/database/bap021.

Zerbino DR, Birney E. 2008. Velvet: algorithms for de novo short read assembly using de Bruijn graphs. Genome Res. 18:821-829. doi:10.1101/gr.074492.107 